\title{
O chá da folha de Morus nigra como agente promotor de qualidade de vida em mulheres na transição menopáusica
}

\author{
The Morus nigra leaf tea as a promoter of quality of life in women in the menopausal \\ transition \\ El té de hoja de Morus nigra como promotor de la calidad de vida en mujeres en la \\ transición menopáusica
}

Sandrine da Silva Miranda ${ }^{1}$, Júlia Lima Gandolfo' ${ }^{1}$, Roane Gabelini Caixeta Vieira ${ }^{1}$, Maria Carolina Alves Zanatta ${ }^{1}$, José Renato Franco Alves ${ }^{2}$, Carlos César Scala de Almeida ${ }^{1}$, Tamara Veiga Faria ${ }^{1 *}$.

\section{RESUMO}

Objetivo: Analisar os efeitos da Morus nigra nos sintomas climatéricos e na qualidade de vida de mulheres na transição menopáusica. Métodos: Trata-se de um estudo clínico, conduzido com 20 mulheres entre 45 e 60 anos que fizeram uso diariamente do chá da folha de Morus nigra por 60 dias e preencheram os questionários Short Form Health Survey, Índice Menopausal de Blatt-Kupperman e Questionário de Saúde da Mulher, a cada 20 dias até completarem o tratamento. Resultados: A soma dos escores do Índice Menopausal de Blatt-Kupperman retratou a melhora da intensidade dos sintomas e, aos 60 dias, não se identificou caso de intensidade acentuada. Os escores totais do Questionário de Saúde da Mulher confirmou a melhora dos sintomas climatéricos no período de estudo $(p<0,001)$, melhora dos sintomas somáticos $(p=0,011)$; vasomotores $(p<0,001)$; problemas de sono $(p=0,024)$; atratividade $(p=0,042)$ e memória $(p=0,045)$. O Short Form Health Survey mostrou a melhora relacionada ao tempo de tratamento $(p<0,001)$ e domínios: capacidade funcional $(p<0,001)$; dor $(p=0,028)$; vitalidade $(0,016)$ e saúde mental $(p=0,035)$. Conclusão: $O$ chá da folha da Morus nigra pode ser uma opção terapêutica capaz de amenizar os sintomas climatéricos, contribuindo para a melhora na qualidade de vida de mulheres nessa fase da vida.

Palavras-chave: Morus, Climatério, Menopausa, Qualidade de vida, Fitoterapia.

\section{ABSTRACT}

Objective: To analyze the effects of Morus nigra on climacteric symptoms and on the quality of life of women in the menopausal transition. Methods: This is a clinical study, conducted with 20 women between 45 and 60 years of age who used Morus nigra leaf tea daily for 60 days and completed the Short Form Health Survey, Blatt-Kupperman Menopausal Index and Questionnaire Women's Health, every 20 days until they complete treatment. Results: The sum of the Blatt-Kupperman Menopausal Index scores shows the improvement of intense symptoms and, at 60 days, did not identify a case of marked intensity. The total scores of the Women's Health Questionnaire confirmed improvement in climacteric symptoms during the study period $(p<0.001)$, improvement in somatic symptoms $(p=0.011)$; vasomotor $(p<0.001)$; sleep problems $(p=0.024)$; attractiveness $(p=0.042)$ and memory $(p=0.045)$. The Short Form Health Survey showed a useful improvement in treatment time $(p<0.001)$ and domains: functional capacity $(p<0.001)$; pain $(p=0.028)$; vitality (0.016) and mental health $(p=0.035)$. Conclusion: The Morus nigra leaf tea may be a therapeutic option capable of alleviating climacteric symptoms, contributing to the improvement in the quality of life of women in this stage of life.

Keywords: Morus, Climacteric, Menopause, Quality of life, Phytotherapy.

\footnotetext{
${ }^{1}$ Faculdade de Medicina Ceres (FACERES), São José do Rio Preto - SP.

*E-mail: tamaraveiga@yahoo.com.br

2 Faculdade de Medicina de Ribeirão Preto da Universidade de São Paulo (FMRP-USP), Ribeirão Preto $\mathrm{SP}$.
} 


\section{RESUMEN}

Objetivo: Analizar los efectos de Morus nigra en los síntomas climatéricos y en la calidad de vida de las mujeres en la transición menopáusica. Métodos: Este es un estudio clínico, realizado con 20 mujeres entre 45 y 60 años que usaron té de hoja de Morus nigra diariamente durante 60 días y completaron la Encuesta de Salud de Formulario Breve, el Índice y el Cuestionario de Menopausia de Blatt-Kupperman Salud de la mujer, cada 20 días hasta que completen el tratamiento. Resultados: La suma de las puntuaciones del índice de menopausia de Blatt-Kupperman muestra la mejora de los síntomas intensos y, a los 60 días, no identificó un caso de marcada intensidad. Las puntuaciones totales del Cuestionario de Salud de la Mujer confirmaron una mejoría en los síntomas climatéricos durante el período de estudio $(p<0.001)$, mejoría en los síntomas somáticos $(p=0.011)$; vasomotor $(p<0,001)$; problemas de sueño $(p=0.024)$; atractivo $(p=0.042)$ y memoria $(p=0.045)$. La Encuesta de Salud de Forma Corta mostró una mejora útil en el tiempo de tratamiento ( $p$ $<0.001)$ y dominios: capacidad funcional $(p<0.001)$; dolor $(p=0.028)$; vitalidad $(0.016)$ y salud mental $(p=$ 0.035). Conclusión: El té de hoja de Morus nigra puede ser una opción terapéutica capaz de aliviar los síntomas climatéricos, contribuyendo a la mejora de la calidad de vida de las mujeres en esta etapa de la vida.

Palabras clave: Morus, Climaterio, Menopausia, Calidad de vida, Fitoterapia.

\section{INTRODUÇÃO}

Os fitoterápicos são produtos obtidos de plantas medicinais e de seus derivados, e possuem intuito de profilaxia, cura ou tratamento paliativo de doenças, se caracterizando por sua eficácia e qualidade (OLIVEIRA ACD e ROPKE CD, 2016). Esses medicamentos constituem uma opção de cuidado que faz parte da cultura popular há milhares de anos, no entanto, nas últimas décadas, o interesse tem crescido significativamente para usuários e pesquisadores (FIUT MA, et al., 2018).

No Brasil, o uso de plantas medicinais está fortemente presente na população e diretrizes do Ministério da Saúde propõem a ampliação das opções terapêuticas aos usuários e a garantia de acesso às plantas medicinais e aos fitoterápicos em serviços relacionados ao Sistema Único de Saúde (SUS). (NASCIMENTO JÚNIOR BJ, et al., 2016). Haja vista que o hábito da utilização de plantas medicinais é favorável a saúde, uma vez que as pessoas saibam sobre suas formas de uso, os riscos e benefícios (ISERHARD, 2009).

Dentre as diversas finalidades de uso das plantas medicinais, incluem-se aquelas empregadas para 0 tratamento da saúde da mulher. Alguns estudos têm apontado que esta prática é usual no Brasil, destacandose o uso de plantas para o tratamento de sintomas e sinais advindos do processo do climatério (SÁ, 2012). O conhecimento dos efeitos colaterais e dos riscos da terapia hormonal (TH) representa uma busca crescente por uma alternativa eficaz e segura para sua substituição, como por exemplo, a utilização de fitoterápicos (ROCHA BMA, et al., 2018; NUNES CMAC, 2019).

Os fitoterápicos utilizados durante o climatério são obtidos de vegetais ricos em fitoestrógenos, um grupo de compostos fitoquímicos que incluem isoflavonas, lignanas, cumestanos e estilbenos, nos quais a atividade farmacológica deriva da semelhança estrutural aos estrógenos, isto é, possui um anel fenólico, pré-requisito para a ligação aos receptores estrogênicos (PATISAUL HB e JEFERSON W, 2010; MOREIRA AC, et al., 2014).

Nesse contexto, apresenta-se a planta Morus nigra, que é uma espécie pertencente ao gênero Morus, da família Moraceae, conhecida como "amora-preta" ou "amora-miúra". A partir das referências de uso popular do chá de suas folhas, cuja eficiência corresponderia ao alívio de manifestações como ondas de calor e de tensão pré-menstrual, supõe-se que os componentes dessas folhas contenham substâncias capazes de exercer algum efeito sobre os sintomas climatéricos (PEREIRA PM, 2018; PADILHA MM, et al., 2010).

Além disso, há a comercialização das folhas de amora seca para diversos fins, tais como diurético, antihipertensivo, hipoglicêmico e, principalmente, para sintomas climatéricos. (MIRANDA MA, et al., 2010). Nesse sentido, diversos estudos e ensaios clínicos têm sido realizados a respeito dos benefícios da fitoterapia em relação aos sintomas da menopausa (FRANCO OH, et al., 2016). 
A Morus nigra se apresenta como uma opção de fitoterapia que oferece às mulheres e aos especialistas a possibilidade de abordagem medicamentosa que poderia contribuir para a melhoria da qualidade de vida (QV) da mulher (FIGUEREDO KC, et al., 2018). Atualmente, considera-se que a QV na transição menopáusica se relaciona tanto com a presença de sintomas decorrentes do hipoestrogenismo quanto com ondas de calor, alterações psicológicas, urogenitais, sexuais e distúrbios do sono, além de fatores culturais referentes ao processo de envelhecimento e à interação entre eles (SOUZA LSA e ARAÚJO LOC, 2015).

A avaliação da QV da mulher durante o climatério passa a ser parte integrante de qualquer tentativa de avaliar o impacto de uma intervenção médica e suas repercussões futuras, uma vez que $96 \%$ das mulheres nessa fase apresentam sintomatologia que afeta sua QV. Ademais, a análise desses fatores torna-se alvo de pesquisas, visto que seus resultados podem ajudar a definir estratégias de promoção da saúde e condutas terapêuticas no sistema de saúde (ANDRADE RL, et al., 2019; SOUZA LSA e ARAÚJO LOC, 2015).

Sendo assim, devido ao crescente interesse pelos efeitos dos fitoterápicos, ao uso o popular e amplo de Morus nigra no Brasil, e à utilização de produtos naturais de baixo custo e de fácil acesso, o presente estudo se propôs a analisar os efeitos do chá feito de suas folhas como agente promotor de QV em mulheres na transição menopáusica.

\section{MÉTODOS}

O estudo foi aprovado pelo Comitê de Ética em Pesquisa da Faculdade Ceres (FACERES), recebendo o número 2.403.622 correspondentes ao parecer de aprovação. Está cadastrado no Registro Brasileiro de Ensaios (ReBEC) sob o número UTN como: U1111-1211-0884. Trata-se de um estudo clínico não randomizado, cuja amostragem é do tipo aleatória simples.

As participantes do estudo foram identificadas durante as consultas de rotinas na Unidade Básica de Saúde, de uma cidade do interior paulista. Foram selecionadas mulheres entre 45 e 60 anos, que apresentassem exames de colpocitologia oncótica e mamografia atualizados, sem alterações ou com alterações benignas, cuja data da última menstruação tivesse sido há, pelo menos, 6 meses e que estivessem apresentando sintomas de privação estrogênica e irregularidade menstrual, podendo ou não estar fazendo uso de terapia hormonal.

Como critérios de exclusão foram considerados: mulheres tabagistas; etilistas com ingesta de álcool superior a um litro por semana; vegetarianas ou macrobióticas; histórico de doenças crônicas gastrointestinais de absorção; histórico de episódios de hipoglicemia.

$\mathrm{Na}$ visita de triagem, as participantes consentiram sua participação no estudo, foram coletados dados para perfil sociodemográfico e em seguida, preencheram os questionários validados no Brasil - Short Form Health Survey (SF-36), Índice Menopausal de Blatt-Kupperman (IMBK) e Questionário de Saúde da Mulher (QSM), os quais foram disponibilizados integralmente na forma virtual de formulário do Google (GoogleForms) e respondidos a cada 20 dias até completarem 60 dias de tratamento.

A avaliação da influência de patologias na QV de forma não específica foi feita pelo uso do questionário SF-36, composto por 36 itens classificados em oito domínios. Avalia tanto os aspectos negativos da saúde (enfermidade), como os aspectos positivos (bem-estar) (CICONELLI RM, 1997).

O QSM, composto por 36 questões, permite a avaliação específica relacionado à menopausa e à mensuração de uma intervenção na QV da mulher (SILVA FILHO CR, et al., 2015). A especificação, quantificação e classificação dos sinais e sintomas do climatério foram realizadas por meio do IMBK. Tratase de um instrumento que envolve 11 queixas permitindo a categorização dos sintomas segundo sua intensidade (MELO CRM, et al., 2016).

No preparo do produto investigacional foram utilizadas folhas de Morus nigra, coletadas em uma cidade do interior paulista, que foram higienizadas, secas à sombra e realizada maceração mecânica, reproduzindo o uso popular. As participantes do estudo receberam os 60 sachês de doses diárias, sendo instruídas quanto ao preparo a partir da infusão obtida por $7 \mathrm{~g}$ de folhas maceradas em $600 \mathrm{ml}$ de água fervida a $90^{\circ} \mathrm{C}$, fazendo o uso de $200 \mathrm{ml}$, três vezes ao dia, podendo ser conservada sob refrigeração por até 24 horas (OLIVEIRA ACB, et al., 2013). 
No acompanhamento das participantes, elas receberam atendimento médico por profissional especializado em ginecologia e tiveram assistência contínua durante e após o término do tratamento, destacando a importância da escuta qualificada paralela às intervenções.

Para a análise estatística foram determinadas médias, desvios-padrão e porcentagens das variáveis estudadas para a caracterização da população estudada. Os dados foram comparados por meio do Teste Friedman e Teste Wilcoxon, com nível de significância de 5\%, utilizando-se o programa SPSS versão 24.0.

\section{RESULTADOS}

Participaram do estudo 20 mulheres na faixa etária entre 45 e 60 anos, com mediana de 52 anos de idade. Elas responderam aos questionários em formulário Google forms conforme agendamento do estudo, sem dificuldades e todas as questões foram completadas.

Houve predomínio de participantes que não faziam uso de TH prévia (90\%) e de mulheres que referiram ter vida sexual ativa (90\%). A irregularidade menstrual esteve presente em $45 \%$ das mulheres, sendo a metade $(50 \%)$ em fase pós-menopáusica.

Considerando a Tabela 1, observa-se que no baseline, a soma dos escores IMBK alcançaram intensidade leve em $25 \%$, moderada em $60 \%$ e acentuada em $15 \%$ das participantes. Não foram notadas pacientes totalmente assintomáticas pela medição dos sintomas por esse instrumento. Nas mensurações subsequentes, os escores retrataram melhora das intensidades e, ao final dos 60 dias, a intensidade leve representou $65 \%$, moderada $35 \%$, sem nenhum caso de intensidade acentuada (Tabela 1 ).

Tabela 1 - Distribuição percentual dos sintomas climatéricos e suas intensidades, segundo o IMBK.

\begin{tabular}{cccccc}
\hline IMBK & $\mathbf{B L}$ & $\mathbf{2 0}$ dias & $\mathbf{4 0}$ dias & $\mathbf{6 0}$ dias & Valor $\boldsymbol{p}$ \\
\hline Leve $(=19)$ & $5(25 \%)$ & $10(50 \%)$ & $12(60 \%)$ & $13(65 \%)$ & $<0,001$ \\
Moderado $(20-35)$ & $12(60 \%)$ & $9(45 \%)$ & $6(30 \%)$ & $7(35 \%)$ & $<0,001$ \\
Acentuado $(>35)$ & $3(15 \%)$ & $1(5 \%)$ & $2(10 \%)$ & $0(0 \%)$ & $<0,001$ \\
\hline
\end{tabular}

Fonte: Miranda SS, et al., 2020.

Na Tabela 2, nota-se a melhora significante dos sintomas mensurados pela IMBK relacionados ao tempo de uso da Morus Nigra, incluindo cada um dos sintomas referidos nesse instrumento. Houve melhora significativa na maioria dos sintomas definidos para o IMBK e que podem ser relacionados à QV das participantes (Tabela 2).

Tabela 2 - Percentagem de mulheres participantes no estudo com sintomas climatéricos e suas intensidades de acordo com o período de tratamento, conforme o IMBK.

\begin{tabular}{|c|c|c|c|c|c|c|c|c|c|c|c|c|c|c|c|c|c|}
\hline \multirow[b]{2}{*}{ Variáveis } & \multicolumn{4}{|c|}{ Baseline } & \multicolumn{4}{|c|}{20 dias } & \multicolumn{4}{|c|}{40 dias } & \multicolumn{4}{|c|}{60 dias } & \multirow[b]{2}{*}{ Valor $p$} \\
\hline & $\begin{array}{l}0 \\
\%\end{array}$ & $\begin{array}{l}1 \\
\%\end{array}$ & $\begin{array}{l}2 \\
\%\end{array}$ & $\begin{array}{l}3 \\
\%\end{array}$ & $\begin{array}{l}0 \\
\%\end{array}$ & $\begin{array}{l}1 \\
\%\end{array}$ & $\begin{array}{l}2 \\
\%\end{array}$ & $\begin{array}{l}3 \\
\%\end{array}$ & $\begin{array}{l}0 \\
\%\end{array}$ & $\begin{array}{l}1 \\
\%\end{array}$ & $\begin{array}{l}2 \\
\%\end{array}$ & $\begin{array}{l}3 \\
\%\end{array}$ & $\begin{array}{l}0 \\
\%\end{array}$ & $\begin{array}{l}1 \\
\%\end{array}$ & $\begin{array}{l}2 \\
\%\end{array}$ & $\begin{array}{l}3 \\
\%\end{array}$ & \\
\hline $\begin{array}{l}\text { Ondas de } \\
\text { calor }\end{array}$ & 5 & 15 & 35 & 45 & 15 & 35 & 35 & 15 & 20 & 50 & 30 & 0 & 20 & 50 & 25 & 5 & $<0,001^{\star}$ \\
\hline Parestesia & 50 & 15 & 20 & 15 & 50 & 20 & 20 & 10 & 50 & 25 & 25 & 0 & 60 & 35 & 5 & 0 & $0,022^{*}$ \\
\hline Insônia & 25 & 20 & 30 & 25 & 35 & 35 & 15 & 15 & 35 & 30 & 25 & 10 & 35 & 30 & 25 & 10 & $0,021^{*}$ \\
\hline Nervosismo & 0 & 35 & 35 & 30 & 10 & 45 & 35 & 10 & 25 & 30 & 45 & 0 & 20 & 50 & 30 & 0 & $<0,001^{*}$ \\
\hline Melancolia & 25 & 35 & 35 & 5 & 30 & 30 & 35 & 5 & 30 & 35 & 25 & 10 & 50 & 35 & 15 & 0 & $0,004^{*}$ \\
\hline Vertigem & 45 & 25 & 25 & 0 & 50 & 40 & 10 & 0 & 55 & 25 & 20 & 0 & 75 & 20 & 5 & 0 & $0,022^{*}$ \\
\hline Fraqueza & 45 & 35 & 20 & 0 & 65 & 20 & 5 & 10 & 60 & 20 & 20 & 0 & 55 & 30 & 10 & 5 & 0,725 \\
\hline $\begin{array}{c}\text { Artralgia/mia } \\
\text { Igia }\end{array}$ & 10 & 25 & 10 & 55 & 20 & 20 & 40 & 20 & 15 & 40 & 25 & 20 & 15 & 40 & 40 & 5 & $0,016^{*}$ \\
\hline Cefaleia & 25 & 25 & 30 & 20 & 45 & 30 & 15 & 10 & 45 & 25 & 25 & 5 & 30 & 50 & 20 & 0 & $0,049^{*}$ \\
\hline Palpitações & 40 & 30 & 25 & 5 & 55 & 15 & 25 & 5 & 60 & 10 & 25 & 5 & 60 & 20 & 20 & 0 & 0,143 \\
\hline $\begin{array}{c}\text { Formigamen } \\
\text { to }\end{array}$ & 50 & 15 & 10 & 25 & 45 & 30 & 15 & 10 & 55 & 20 & 20 & 5 & 65 & 20 & 15 & 0 & 0,072 \\
\hline
\end{tabular}

Legenda: 0: ausente; 1: intensidade leve; 2: intensidade moderada; 3: intensidade acentuada; ${ }^{*}$ valor $p$ indicando significância estatística. Fonte: Miranda SS, et al., 2020. 
Nesse mesmo instrumento, avaliou-se o número de ondas de calor durante o dia, conforme representado pela Tabela 3, na qual se constata diminuição geral relevante do número de ondas de calor com $p=0,000$, especificamente nos períodos de 20, 40 e 60 dias em relação ao baseline, assim como significante redução entre 20 e 40 dias, não apresentando resposta comprovada apenas entre 40 e 60 dias. Ademais, foi determinado aumento de participantes com ausência desse sintoma (Tabela 3).

Tabela 3 - Número de ondas de calor por dia referido por participantes (frequência), distribuídas no período de acompanhamento do estudo.

\begin{tabular}{cc|cc|cc|cc}
\hline \multicolumn{2}{c|}{ Baseline } & \multicolumn{2}{c|}{20 dias } & \multicolumn{2}{c|}{$\mathbf{4 0}$ dias } & \multicolumn{2}{c}{$\mathbf{6 0}$ dias } \\
$\mathbf{N}^{\mathbf{0}}$ ondas & Frequência & \multicolumn{2}{|c|}{$\mathbf{N}^{\mathbf{0}}$ ondas Frequência } & $\mathbf{N}^{\mathbf{0}}$ ondas & Frequência & $\mathbf{N}^{\mathbf{0}}$ ondas & Frequência \\
\hline 0 & 1 & 0 & 4 & 0 & 6 & 0 & 10 \\
1 & 1 & 1 & 2 & 1 & 1 & 1 & 3 \\
2 & 3 & 2 & 3 & 2 & 8 & 2 & 6 \\
4 & 3 & 3 & 5 & 3 & 1 & 3 & 1 \\
5 & 6 & 4 & 1 & 4 & 2 & - & - \\
6 & 1 & 5 & 3 & 5 & 1 & - & - \\
7 & 2 & 6 & 1 & 6 & 1 & - & - \\
8 & 2 & 8 & 1 & - & - & - & - \\
10 & 1 & - & - & - & - & - & - \\
\hline
\end{tabular}

Legenda: Frequência: representa o número de mulheres que referiram ondas de calor; $\mathrm{N}^{\circ}$ ondas: número de ondas de calor por dia.

Fonte: Miranda SS, et al., 2020.

Na Tabela 4, pode-se observar na comparação dos escores totais de cada período e cada componente do QSM, a melhora estatisticamente significante dos sintomas climatéricos no período de 60 dias $(\mathrm{p}<0,001)$. A melhora gradativa desses sintomas pode ser ressaltada pelas comparações dos períodos baseline com 20 $(p=0,001), 40(p=0,008)$ e 60 dias $(p=0,002)$. Houve significância clínica dos sintomas somáticos $(p=0,011)$; memória $(p=0,045)$; sintomas vasomotores $(p<0,001)$; problemas de sono $(p=0,024)$ e atratividade $(p=0,042)$. O componente "depressão" $(p=0,078)$ apresenta uma tendência estatística de melhora (Tabela 4).

Tabela 4 - Classificação dos domínios do QSM relacionados ao período de acompanhamento do estudo.

\begin{tabular}{cccccc}
\hline Domínio & Baseline & 20 dias & $\mathbf{4 0}$ dias & $\mathbf{6 0}$ dias & Valor $\mathbf{p}$ \\
\hline Depressão & 13,900 & 13,100 & 13,000 & 11,850 & 0,078 \\
Sintomas somáticos & 18,050 & 15,950 & 16,700 & 15,550 & $0,011^{*}$ \\
Memória & 8,750 & 7,800 & 7,800 & 7,700 & $0,045^{\star}$ \\
Sintomas vasomotores & 6,750 & 5,500 & 4,750 & 4,500 & $<0,0001^{*}$ \\
Ansiedade/temores & 8,600 & 7,550 & 7,600 & 7,550 & 0,227 \\
Comportamento Sexual & 8,750 & 8,500 & 8,150 & 7,800 & 0,243 \\
Problemas no sono & 8,650 & 7,600 & 7,450 & 7,560 & $0,024^{*}$ \\
Sintomas menstruais & 8,400 & 7,150 & 7,350 & 7,250 & 0,160 \\
Atratividade & 8,750 & 8,500 & 8,150 & 7,800 & $0,042^{*}$ \\
\hline Total & 90,500 & 83,000 & 76,500 & 75,000 & $<0,001^{*}$ \\
\hline
\end{tabular}

Fonte: Miranda SS, et al., 2020.

A análise individual de alguns sintomas, evidenciou melhora da sudorese noturna $(p=0,005)$, assim como do interesse sexual $(p=0,048)$ e da secura vaginal $(p=0,005)$. Por outro lado, conforme análise individual, verificou-se que não houve relação entre o uso do chá e o aumento de mastalgia $(p=0,092)$, presença de náuseas e vômitos $(p=0,582)$ e/ou alterações no apetite $(p=0,229)$. 
Com base na Tabela 5, observa-se que o tempo de uso repercutiu em menor escore $(p<0,001)$, representando uma melhora significativa dos sintomas no período de 60 dias. As melhorias, relacionadas ao tempo de uso do produto investigacional, foram identificadas na análise dos domínios: capacidade funcional $(p<0,001)$; dor $(p=0,028)$; vitalidade $(0,016)$ e saúde mental $(p=0,035)$. No domínio "aspectos emocionais" $(p=0,073)$ há uma tendência à diferença estatística demonstrando possível melhora desse domínio relacionado com o tempo de uso (Tabela 5).

Tabela 5 - Relação entre os domínios do questionário SF-36 e o período de tratamento.

\begin{tabular}{cccccc}
\hline Domínio & BL & 20 dias & 40 dias & 60 dias & valor $\boldsymbol{p}$ \\
\hline Capacidade Funcional & 45,000 & 60,000 & 75,000 & 72,500 & $0,001^{*}$ \\
Aspectos físicos & 87,500 & 75,000 & 100,000 & 100,000 & 0,678 \\
Dor & 51,000 & 62,000 & 61,500 & 62,000 & $0,028^{*}$ \\
Estado Geral de saúde & 62,000 & 57,000 & 62,000 & 62,000 & 0,301 \\
Vitalidade & 50,000 & 65,000 & 67,500 & 67,500 & $0,016^{\star}$ \\
Aspectos sociais & 75,000 & 93,750 & 87,500 & 93,750 & 0,175 \\
Aspectos emocionais & 83,350 & 100,000 & 66,700 & 100,000 & 0,073 \\
Saúde Mental & 66,000 & 70,000 & 72,000 & 78,000 & $0,035^{*}$ \\
\hline Total & 90,000 & 81,100 & 80,300 & 76,750 & $<0,001$ \\
\hline
\end{tabular}

Legenda: Comparação de BL: baseline, 20 dias, 40 dias, 60 dias; *valor $p$ estatisticamente significante $<0,05$.

Fonte: Miranda SS, et al., 2020.

Complementarmente, evidencia-se que a melhora dos sintomas ocorre frequentemente no período de baseline correspondente a 20, 40 e 60 dias, sendo que, em sua maioria, há uma estabilização dos sintomas entre os períodos de 40 e 60 dias (Tabela 6).

Tabela 6 - Comparação entre os escores dos domínios do questionário SF-36 e os períodos de realização do estudo.

\begin{tabular}{ccccccc}
\hline Domínio & BL x 20 (p) & BL x 40 (p) & BL x 60 & $\mathbf{2 0 ~ \times ~ 4 0 ~}$ & $\mathbf{2 0 \times 6 0}$ & $\mathbf{4 0} \times \mathbf{6 0}$ \\
\hline Capacidade funcional $(p)$ & $<0,001$ & $<0,001$ & $<0,001$ & 0,045 & 0,753 & 0,361 \\
Dor $(p)$ & 0,015 & 0,033 & 0,017 & 0,038 & 0,345 & 0,864 \\
Vitalidade $(p)$ & 0,020 & 0,025 & 0,008 & 0,024 & 0,105 & 0,076 \\
Saúde mental $(p)$ & 0,076 & 0,078 & 0,003 & 0,051 & 0,074 & 0,064
\end{tabular}

Legenda: valor $p$ resultante da comparação do BL: baseline, 20 dias, 40 dias e 60 dias.

Fonte: Miranda SS, et al., 2020.

\section{DISCUSSÃO}

O consumo do chá das folhas de Morus nigra por mulheres na transição menopáusica demostrou ser eficaz na melhora da qualidade de vida global e no alívio dos sintomas climatéricos, corroborando com a literatura e censo popular. A melhora significante nos escores médios obtidos a partir dos questionários SF36, IMBK e QSM comprova os benefícios oriundos do chá da folha da amora, em 60 dias de uso, ressaltandose que após 40 dias a maioria dos critérios que apresentaram melhora permaneceram estáveis, salvo sintomas vasomotores que continuaram diminuindo.

Os domínios "capacidade funcional" e "vitalidade" do questionário SF-36 retratam a melhora na realização de atividades diárias das participantes devido ao seu estado de saúde. A vitalidade avaliada, engloba quatro itens: vigor, energia, esgotamento e cansaço, sintomas que se relacionam com o domínio "saúde mental" que também apresentou melhora. Esse resultado condiz com o ensaio clínico que utilizou $250 \mathrm{mg}$ de folha de Morus nigra em pó por 60 dias, que também relatou melhora nesses três domínios (COSTA JPL, et al., 2020). 
O domínio "saúde mental" do SF-36, analisa a presença de angústia e bem-estar psicológico, que podem estar relacionados aos sintomas climatéricos. Adicionalmente, na análise do IMBK, houve redução na intensidade dos sintomas de nervosismo e melancolia, o que demostra que a Morus nigra pode intervir no alívio de queixas relacionadas ao humor das mulheres no climatério.

Esse mesmo efeito também foi evidente em estudos de TH, os quais indicaram que o estado do humor parece não só ser decorrente do alívio dos sintomas vasomotores, mas também estar relacionado a um efeito direto dos hormônios sexuais femininos sobre o sistema nervoso central (ZAHAR EV, et al., 2015). Portanto, a concordância da melhora da "saúde mental" simultânea com a "vitalidade" justifica a melhora da QV das mulheres, visto que maior ansiedade, nervosismo e depressão contribuem quanto ao nível de energia e fadiga avaliados na "vitalidade".

O domínio "dor" do SF-36 avalia quanta dor o indivíduo sentiu nas últimas quatro semanas e as limitações que ela provocou em seu cotidiano (ZAHAR EV, et al., 2015; MIRANDA JS, et al., 2014). Apesar de não ser um sintoma específico dessa fase da vida das mulheres, a melhora nessa dimensão contribuiu para a QV em geral, influenciando outros domínios. Além disso, tal resultado ressalta a possível atividade antinociceptiva e anti-inflamatória da Morus nigra, como é demonstrado em outros estudos, podendo ser uma opção de terapia complementar para a dismenorreia e outras dores (PADILHA MM, et al., 2010; SOUZA GR, et al., 2015; BOTAN AG, 2018).

Esses resultados podem ser reafirmados pelos sintomas artralgia/mialgia, cefaleia e parestesia, mensurados pelo IMBK, o qual demonstrou diminuição desses sintomas no período de 60 dias e também melhora no domínio "sintomas somáticos" do QSM.

O QSM é capaz de avaliar, de forma individual e específica, alguns aspectos da QV, sendo mais sensível na detecção de alterações após uma intervenção (HUNTER MS, 2003). A sua aplicação neste estudo agrega relevância devido à qualidade de avaliação desse questionário e aos resultados obtidos a partir dele, haja vista que revelou melhora significativa no escore global de QV e nos seguintes domínios: sintomas somáticos, memória, sintomas vasomotores, problemas no sono e atratividade.

O climatério é a fase na qual as mulheres se tornam mais sensíveis e vulneráveis a diversos sintomas, em razão de fatores metabólicos, fisiológicos, psicológicos e sociais. Sintomas somáticos, como cefaleia, artralgia/mialgia, indisposição e vertigem, são considerados de curto e médio prazo, podendo estar relacionados àqueles de longo prazo, como osteoporose e doenças cardiovasculares (ALENCAR CA, 2015). Dessa forma, podemos considerar que a Morus nigra ao beneficiar os sintomas somáticos, por consequência, otimiza a QV das mulheres que fazem seu uso.

Nesse mesmo sentido, a atratividade refere-se à avaliação e à elaboração de um autoconceito, influenciada por parâmetros físicos e emocionais. A percepção das mulheres a respeito de seu corpo e de sua atratividade se mostra relevante na construção da autoimagem e, portanto, da autoestima global, o que influencia a saúde física, mental e emocional, além das relações sociais e familiares (PARDINI D, 2014).

Outro importante dado que a presente pesquisa propõe é a ação da Morus nigra na melhora da memória. As funções cognitivas - notadamente, a memória - recebem influência do estrogênio, o que explicaria sua piora durante o climatério. Em contrapartida, o estrógeno proveniente da TH não evidencia melhora da memória e não está indicado para a prevenção primária ou secundária da demência, sendo a Morus nigra uma possível alternativa (PARDINI D, 2014).

Neste estudo, o benefício no domínio "sono" que avalia insônia e agitação também se destaca como resultado importante. A presença de transtornos do sono acomete cerca de $15 \%$ da população em geral, e desses, as mulheres são as mais acometidas (30 a 80\%), havendo maior incidência no grupo pós-menopausa, entre 28 e 63\% (SOARES CN, 2006; HACHUL DE CAMPOS H, et al., 2006).

As principais queixas de insônia foram relacionadas a fogachos e a suores noturnos, pois ao ocorrerem durante a noite provocam alterações do sono com consequente fadiga, irritabilidade e desconforto físico (LLANAS AC, et al., 2008). 
Durante o climatério e a pós-menopausa, os sintomas vasomotores, tais como ondas de calor e suores noturnos, são os mais relatados e os que geram maiores repercussões sobre a QV das mulheres. A literatura aponta que as ondas de calor podem desencadear outros constituintes da síndrome climatérica, e correspondem a uma das principais causas de busca por serviços médicos (SANTOS- SÁ D, et al., 2006).

Assim, a melhora com o uso do chá das folhas de Morus nigra nos domínios "problemas do sono" e dos "sintomas vasomotores" condiz com a literatura, pois mostra a interação dos sintomas e assim, a otimização e complementaridade na QV das mulheres, corroborando com a melhora global na avaliação geral do QSM. Os estudos sobre a TH apresentaram resultados positivos relacionados aos sintomas climatéricos, entretanto não relataram benefícios na QV global (MARTINS MAD, et al., 2009).

As maiores contribuições do presente estudo estão relacionadas à comprovação científica da melhora na QV das mulheres e sua relação com os benefícios relacionados aos sintomas vasomotores, confirmada pelos três instrumentos metodológicos. Por meio do questionário IMBK, foi evidenciada diminuição relevante da intensidade e da média de ondas de calor em um dia. Os resultados revelaram que ao início do estudo apenas uma participante referiu ausência do sintoma, e após 60 dias do uso do chá da Morus nigra foi retratada ausência total de ondas de calor em 10 mulheres. Os resultados representam uma diminuição significante no número de ondas de calor nos períodos de 20, 40 e 60 dias quando comparados ao questionário base.

Além disso, o IMBK demonstrou redução da ocorrência de outros sintomas, como: insônia, nervosismo, melancolia, parestesia, vertigem, artralgia/mialgia e cefaleia. No estudo clínico não randomizado que utilizou o extrato hidroalcoólico de Morus nigra administrado em gotas, constatou diminuição das ondas de calor, insônia, cefaleia e fadiga (PEREIRA PM, 2018). O estudo clínico randomizado com administração de cápsulas da folha em pó também apresentou resultado nos mesmos sintomas, acrescido de melhora nos sintomas parestesia e nervosismo. Por outro lado, no presente estudo, o único sintoma que não apresentou redução, divergindo de ambos os estudos clínicos, foi a fadiga. Contudo, o sintoma apresentou melhora na análise do questionário SF-36 no domínio "vitalidade".

$\mathrm{Na}$ avaliação do QSM não foram identificadas mudanças nos domínios "comportamento sexual" e "sintomas menstruais". Entretanto, é válido ressaltar que o uso da Morus Nigra, por pelo menos 60 dias, apresentou melhora significativa da secura vaginal e do interesse sexual. O IMBK, embora seja largamente utilizado por ginecologistas e por estudos científicos como método para aferição dos sintomas climatéricos, apresenta uma limitação por não contemplar sintomas importantes, como os urogenitais e sexuais.

O uso de questionários específicos para avaliação do índice de função sexual pode servir como suporte para futuros estudos, tendo em vista que outros fitoterápicos evidenciam esse benefício, sendo que a suplementação dietética de soja melhorou significativamente a secura vaginal (CARMIGNANI LO, et al., 2010).

A partir dos resultados descritos acima e da ausência de eventos adversos, a Morus nigra pode se apresentar como uma opção terapêutica, tendo uma proporção de melhora dos sintomas climatéricos semelhante a TH. Além disso, ressalta-se que aproximadamente $70 \%$ das mulheres cessam a TH após 0 primeiro ano devido a queixa de mastalgia, náuseas, cefaleia, ganho de peso e retenção hídrica, tais sintomas que não foram manifestados pelas participantes em nosso estudo (GLAZIER MG e BOWMAN MA, 2001). A terapêutica com Morus nigra por meio do extrato hidroalcoólico das folhas e do pó das folhas também não apresentou reações adversas (PEREIRA PM, 2018; COSTA JPL, et al., 2020).

A folha de Morus nigra é considerada de baixa toxicidade por diversos estudos pré-clínicos e evidencia efeito hepatoprotetor demonstrado contra a injúria hepática induzida pelo paracetamol em camundongos (ALMEIDA JRGS, et al., 2011; OLIVEIRA ACB, et al., 2013; BOTAN AG, 2018; MALLHI TH, et al., 2014).

Inclusive, recente estudo demonstrou que a Morus nigra além de oferecer hepatoproteção, também apresenta ação terapêutica na sobrecarga hepática de ferro, com melhoria nas complicações da patologia (FIGUEREDO KC, 2017). Ademais, o estudo clínico com o pó das folhas em cápsulas não apresentou alterações clínicas e laboratoriais das funções hepáticas e renais, na dose diária de $250 \mathrm{mg}$, corroborando com o proposto (COSTA JPL, et al., 2020). 
Vale ressaltar que além de ser de fácil acesso e baixo custo, segundo a Embrapa (Empresa Brasileira de Pesquisa Agropecuária), o cultivo da amoreira-preta é baseado no sistema de produção de base ecológica, ou seja, sem o uso de agroquímicos, o que facilita a manutenção do cultivo e não traz riscos de toxicidade (PAGOT E, et al., 2007).

Os formatos eletrônicos adotados pelo estudo para os questionários de QV facilitam a coleta de dados e contribuem para o cumprimento dos agendamentos do estudo e qualificação do banco de dados. O formulário virtual apresenta várias facilidades em relação aos questionários elaborados em formulário de papel, como facilidade de compilação e processamento automático dos dados coletados. A sua utilização é processo inovador e tão eficaz quanto às entrevistas tradicionais (OLIVEIRA MOR, et al., 2009).

\section{CONCLUSÃO}

O consumo diário do chá feito com $7 \mathrm{~g}$ das folhas de Morus nigra durante 60 dias evidenciou eficácia relacionada à melhora da qualidade de vida das mulheres na transição menopáusica, a partir da amenização dos sintomas climatéricos, incluindo qualidade do sono, atratividade, capacidade funcional, vitalidade, saúde mental, memória e, principalmente, mensuração e intensidade das ondas de calor, além dos benefícios relatados quanto à dor e aos sintomas somáticos. Assim, a Morus nigra pode representar uma opção terapêutica no processo do climatério, baseado nos resultados advindos de seu uso e na escassez de eventos adversos, retratados neste estudo.

\section{REFERÊNCIAS}

1. ALENCAR CA. Sintomas do climatério: prevalência em mulheres de uma unidade de saúde da família do município de Cajazeiras- PB. Monografia (Graduação) - UFCG/CFP. Cajazeiras (PB): Universidade Federal de Campina Grande; 2015.

2. ANDRADE RL, et al. Avaliação da qualidade de vida de mulheres climatéricas atendidas em ambulatório especializado. Brazilian Journal Health, 2019; 2(1): 66-90.

3. BOTAN AG. Citotoxicidade e ação anti-inflamatória in vitro dos extratos glicólicos de Morus nigra (amora), Ziziphus joazeiro (juá) e Vitis vinifera (uva). Dissertação (Mestrado em Biopatologia Bucal) - Pós-graduação em Biopatologia Bucal - Universidade Estadual Paulista (Unesp), Instituto de Ciência e Tecnologia, São José dos Campos, 2018.

4. CAMPOS HH, et al. Sleep disturbances, oxidative stress and cardiovascular risk parameters in postmenopausal women complaining of insomnia. Climacteric, 2006; 9(4): 312-319.

5. CARMIGNANI LO, et al. The effect of dietary soy supplementation compared to estrogen and placebo on menopausal symptoms: A randomized controlled trial. Maturitas, 2010; 67(3): 262-269.

6. CICONELLI RM. Tradução para o português e validação do questionário genérico de avaliação de qualidade de vida "Medical Outcomes Study 36-item short-form health survey (SF-36)". Tese (doutorado) - Universidade Federal de São Paulo (EPM), São Paulo, SP; 1997.

7. COSTA JPL, et al. Randomized double- blind placebo- controlled trial of the effect of Morus nigra L. (black mulberry) leaf powder on symptoms and quality of life among climacteric women. Int J Gynaecol Obstet. 2020; 148(2): $243-252$.

8. FIGUEREDO KC, et al. Safety assessment of Morus nigra L. leaves: Acute and subacute oral toxicity studies in Wistar rats. J Ethnopharmacol, 2018; 224(1): 290- 296.

9. FIGUEREDO KC. Avaliação do perfil fitoquímico, toxicidade e comportamento do extrato das folhas de morus nigra linnaeus frente à sobrecarga de ferro. Dissertação (mestrado) - Universidade Federal de Santa Maria, Centro de Ciências da Saúde, Programa de Pós-Graduação em Ciências Farmacêuticas. Santa Maria, RS, 2017.

10. FIUT MA, et al. A prática clínica em fitoterapia magistral: uma experiência interprofissional da Associação Brasileira de Fitoterapia. Vittalle. Revista de Ciências da Saúde, 2018; 30(1): 152-158.

11. FRANCO OH, et al. Use of plant-based therapies and menopausal symptoms: a systematic review and meta-analysis. JAMA. 2016; 315(23): 2554-63.

12. GLAZIER MG, BOWMAN MA. A review of the evidence for the use of phytoestrogens as a replacement for traditional estrogen replacement therapy. Arch Intern Med. 2001; 161(9): 1161-1172.

13. HUNTER MS. The Women's Health Questionnaire (WHQ): Frequently Asked Questions (FAQ). Health and Quality of Life Outcomes. 2003; 1(41): 1-5.

14. ISERHARD ARM, et al. Práticas culturais de cuidados de mulheres mães de recém-nascido de risco do Sul do Brasil. Escola Anna Nery Revista de Enfermagem. 2009; 13(1): 116-122.

15. LLANAS AC, et al. Physical therapy reduces insomnia symptoms in postmenopausal women. Maturitas, 2008; 61(3): 281-284.

16. MALLHI TH, et al. Hepatoprotective activity of aqueous methanolic extract of Morus nigra against paracetamol-induced hepatotoxicity in mice. Bangladesh Journal of Pharmacology, 2014; 9(1): 60-66.

17. MARTINS MAD, et al. Qualidade de vida em mulheres na pós-menopausa, usuárias e não usuárias de terapia hormonal. Revista Brasileira de Ginecologia e Obstetrícia. 2009; 31(4): 196-202. 
18. MELO CRM, et al. Aplicação do Índice Menopausal de kupperman: um estudo transversal com mulheres climatéricas. Espaço para a Saúde - Revista de Saúde Pública do Paraná. 2016; 17(2): 41-50.

19. MIRANDA JS, et al. Quality of life of postmenopausal women attended at Primary Health Care. Revista Brasileira Enfermagem. 2014; 67(5): 803-9

20. MIRANDA MA, et al. Uso etnomedicinal do chá de Morus nigra L. no tratamento dos sintomas do climatério de mulheres de Muriaé, Minas Gerais, Brasil. HU Revista, Juiz de Fora, 2010; 36(1): 61-68.

21. MOREIRA AC, et al. Phytoestrogens as alternative hormone replacement therapy in menopause: What is real, what is unknown. Journal of Steroid Biochemistry and Molecular Biology. 2014; 143(1): 61-71.

22. NASCIMENTO JÚNIOR BJ, et al. Avaliação do conhecimento e percepção dos profissionais da estratégia de saúde da família sobre o uso de plantas medicinais e fitoterapia em Petrolina-PE, Brasil. Revista Brasileira Plantas Medicinais, 2016; 18(1): 57-66.

23. NUNES CMAC. Conhecimento popular sobre plantas medicinais para o tratamento de sintomas climatéricos em Ouro Preto, Minas Gerais. Monografia (Graduação em Farmácia) - Escola de Farmácia, Universidade Federal de Ouro Preto, Ouro Preto; 2019.

24. OLIVEIRA ACB, et al. Avaliação toxicológica pré-clínica do chá das folhas de Morus nigra L. (Moraceae). Revista brasileira de plantas medicinais, 2013; 15(2): 244-249.

25. OLIVEIRA ACD, ROPKE CD. Os dez anos da Política Nacional de Plantas Medicinais e Fitoterápicos (PNPMF) e os principais entraves da cadeia produtiva de extratos vegetais e medicamentos fitoterápicos no Brasil. Revista Fitos, 2016; 10(2): 95-219.

26. OLIVEIRA MOR, et al. Uma comparação entre entrevistas face to face e entrevistas on-line via chat, aplicando-se a técnica ladderin. Gestão \& Regionalidade. 2009; 25(75): 57-72.

27. PADILHA MM, et al. Estudo farmacobotânico das folhas de amoreira-preta, Morus nigra L., Moraceae. Revista Brasileira Farmacogn. 2010; 20(4): 621-626.

28. PAGOT E, et al. Cultivo de amora-preta. Bento Gonçalves: Embrapa Uva e Vinho. Circular Técnica. 2007; 75(1): 1-11.

29. PARDINI D. Terapia de reposição hormonal na menopausa. Arquivos Brasileiros de Endocrinologia \& Metabologia. 2014; 58(2): 172-181.

30. PATISAUL HB, JEFFERSON W. The pros and cons of phytoestrogens. Frontiers in Neuroendocrinolog. 2010; 3(4): 400-419.

31. PEREIRA PM. Aplicabilidade do extrato hidroalcoolico de amora miura (Morus nigra L.) em mulheres no climatério. Trabalho de Conclusão de Curso (Graduação em Farmácia) - Universidade Estadual da Paraíba, Campina Grande, 2018.

32. ROCHA BMA, et al. Terapias Complementares: fitoterapia como opção terapêutica no climatério e menopausa. Rev. Ciências da Saúde Nova Esperança. 2018; 16(1): 16-25.

33. SÁ IM. "Fito-hormônios": ciência e natureza no tratamento do climatério. Physis Revista de Saúde Coletiva, 2012; 22(4): 1503-1522.

34. SANTOS-SÁ D, et al. Fatores associados à intensidade das ondas de calor em mulheres em climatério. Revista da Associação Médica Brasileira, 2006; 52(6): 413-418.

35. SILVA FILHO CR, et al. Climacteric symptoms and quality of life: validity of women's health questionnaire. Revista Saúde Pública. 2015; 39(3): 333-339.

36. SOARES CN. Insônia na menopausa e perimenopausa: características clínicas e opções terapêuticas. Revista Psiquiatria Clínica 2006; 33(2): 103-109.

37. SOUZA GR, et al. Atividade antinociceptiva do extrato etanólico das folhas de Morus nigra L. (Moraceae). Revista de Ciências Farmacêuticas Básica e Aplicada, 2015; 36(1): 137-142.

38. SOUZA LSA, ARAÚJO LOC. Marco do envelhecimento feminino, a menopausa: sua vivência, em uma revisão de literatura. Revista Kairós Gerontologia. 2015; 18(2): 149-165.

39. ZAHAR EV, et al. Qualidade de vida em usuárias e não usuárias de terapia de reposição hormonal. Revista da Associação Medica Brasileira, 2015; 51(3): 133-138. 